OPEN ACCESS

Edited by:

Ran Wang,

Beijing Academy of Agricultural and

Forestry Sciences, China

Reviewed by:

Wenwu Zhou,

Zhejiang University, China

Hong-xing $X u$,

Shaanxi Normal University, China

${ }^{*}$ Correspondence:

Guo-Qing L

ligq@njau.edu.cn

Specialty section

This article was submitted to Invertebrate Physiology, a section of the journal

Frontiers in Physiology

Received: 12 July 2021

Accepted: 16 August 2021

Published: 30 September 2021

Citation:

Kang $W-N$, Jin L, Ma H-Y and Li G-Q

(2021) Integrated

Microbiome-Metabolome Analysis Reveals Stage-Dependent Alterations in Bacterial Degradation of Aromatics

in Leptinotarsa decemlineata.

Front. Physiol. 12:739800.

doi: 10.3389/fphys.2021.739800

\section{Integrated Microbiome-Metabolome Analysis Reveals Stage-Dependent Alterations in Bacterial Degradation of Aromatics in Leptinotarsa decemlineata}

\author{
Wei-Nan Kang ${ }^{1}$, Lin Jin ${ }^{1}$, Hong-Yu Ma ${ }^{2}$ and Guo-Qing Li ${ }^{1 *}$ \\ ${ }^{1}$ Education Ministry Key Laboratory of Integrated Management of Crop Diseases and Pests, College of Plant Protection, \\ Nanjing Agricultural University, Nanjing, China, ${ }^{2}$ Public Laboratory Platform, College of Plant Protection, Nanjing Agricultural \\ University, Naniing, China
}

To avoid potential harm during pupation, the Colorado potato beetle Leptinotarsa decemlineata lives in two different habitats throughout its developmental excursion, with the larva and adult settling on potato plants and the pupa in soil. Potato plants and agricultural soil contain a specific subset of aromatics. In the present study, we intended to determine whether the stage-specific bacterial flora plays a role in the catabolism of aromatics in L. decemlineata. Kyoto Encyclopedia of Genes and Genomes (KEGG) pathway analysis of the operational taxonomic units (OTUs) obtained by sequencing of culture-independent $16 \mathrm{~S}$ rRNA region enriched a group of bacterial genes involved in the elimination of mono- and polycyclic aromatics at the pupal stage compared with those at the larval and adult periods. Consistently, metabolome analysis revealed that dozens of monoaromatics such as styrene, benzoates, and phenols, polycyclic aromatics, for instance, naphthalene and steroids, were more abundant in the pupal sample. Moreover, a total of seven active pathways were uncovered in the pupal specimen. These ways were associated with the biodegradation of benzoate, 4-methoxybenzoate, fluorobenzoates, styrene, vanillin, benzamide, and naphthalene. In addition, the metabolomic profiles and the catabolism abilities were significantly different in the pupae where their bacteria were removed by a mixture of three antibiotics. Therefore, our data suggested the stage-dependent alterations in bacterial breakdown of aromatics in L. decemlineata.

Keywords: Leptinotarsa decemlineata, stage-specific bacteria, habitat, aromatics, biodegradation

\section{INTRODUCTION}

For holometabolous insect species, sessile pupae are defenseless against potentially harmful factors such as pathogen infection, parasitism, predation, and desiccation. Consequently, a lot of Holometabolans leave their host plants and pupate in soil, an adaptation termed as ontogenetic niche shift (ONS). For example, the final instar larval period of the Colorado potato beetle Leptinotarsa decemlineata is divided into two subphases, the feeding and wandering stages. Whereas, a feeding beetle continuously gnaws potato foliage, a wandering larva typically undergoes an ONS to pupate in the soil (Meng et al., 2019). Obviously, a L. decemlineata beetle lives in two 
different habitats throughout its developmental excursion, with the larva and adult settling on potato plants and the pupa in soil.

In the present paper, we focused on a serious challenge for $L$. decemlineata: degrading excessive aromatics derived from potato plants and agricultural soil. In potato plants, the metabolism of three aromatic amino acids (i.e., tyrosine, phenylalanine, and tryptophan) can produce large number of monoaromatics. These monoaromatics can be used to biosynthesize structurally complex substances. For instance, catecholamines, derived from tyrosine and phenylalanine, can be used to produce betalains, alkaloids, melanins, and hydroxycinnamic acid amides (GandiaHerrero and Garcia-Carmona, 2013; Kostyn et al., 2020). To improve soil quality and increase crop yield, the plant residues, such as straws and stubbles after harvest, organic fertilizers, for example, manure and human excreta, and other organic landfills, are often applied to agricultural fields. These additives generate a variety of organic compounds incorporated into agricultural soil (Kumar and Goh, 2000; Hanselman et al., 2003; Kjaer et al., 2007). Among these organic pollutants are monoaromatics, for instance, benzene, toluene, ethylbenzene, xylene (collectively known as BTEX), styrene, and phenol and polycyclic aromatics such as naphthalene, dioxin, and steroids (Chen et al., 2017; Steinmetz et al., 2019).

Some aromatics are toxic, antinutritive, or/and repellent at high concentrations (Ceja-Navarro et al., 2015; Hammer and Bowers, 2015; Vilanova et al., 2016; Berasategui et al., 2017). Specifically, catecholamines are toxic and can pose a threat to cellular components (Kostyn et al., 2020). Moreover, quinones can lead to the formation of quinoprotein by a reaction between dopaquinone and the sulfhydryl groups of proteins and cause negative effects such as enzyme deactivation, mitochondrial dysfunction, DNA fragmentation, and apoptosis (Mushtaq et al., 2013; Kostyn et al., 2020). Furthermore, dopa may be incorporated into proteins via mimicking tyrosine or phenylalanine in the respective tRNA synthesis (Rodgers and Shiozawa, 2008), or serves as a deterrent to herbivores (Fürstenberg-Hägg et al., 2013; Kostyn et al., 2020). Therefore, these aromatics may adversely affect $L$. decemlineata when accumulated to high concentrations in the body.

It is well-known that insects have evolved different mechanisms to circumvent deleterious effects from the environment (Berasategui et al., 2017). One of the strategies is enlisting the cooperation of bacteria (Lesperance and Broderick, 2020). These bacteria dramatically change during development (Chen et al., 2016; Kang et al., 2021). For instance, a shift of microbial flora coupled with ONS has been documented in $L$. decemlineata, where a total of 18 bacteria genera are specifically distributed in pupae. In contrast, a subset of bacteria genera has larger populations in larvae and adults than those in pupae (Kang et al., 2021). Accordingly, we hypothesized that the stage-specific bacteria flora forms different symbiotic interplays with $L$. decemlineata to biodegrade stage-dependent aromatics from potato plant and agricultural soil, respectively.

The objective of our study was to test the hypothesis. First, we found that a group of bacterial genes involved in the catabolism of monoaromatics and polycyclic aromatics were abundantly expressed at the pupal stages. Second, we evaluated the metabolome profiles in the fourth-instar larvae, pupae, and adults by ultra-performance liquid chromatographyquadrupole-time of flight mass spectrometry (UPLC-Q-TOF MS). We identified stage-specific aromatic biomarkers and active pathways. Finally, we removed bacteria in the pupae by a mixture of three antibiotics, and compared the metabolome profiles between control and treated pupae. Our results revealed stagedependent alterations in the bacterial breakdown of aromatics in L. decemlineata. We argue that the larvae and adults rely on two routes to deal with excessive aromatics, namely, bacterial biodegradation and intestinal excretion. In contrast, the pupae mainly depend on bacteria to catabolize aromatics since the alimentary canal is not well-developed.

\section{METHODS AND MATERIALS}

\section{Insect Rearing and Sampling}

The $L$. decemlineata beetles were routinely reared using a previously described method (Meng et al., 2019). Briefly, the beetles were maintained in an insectary at $28^{\circ} \mathrm{C}$, under a $16: 8 \mathrm{~h}$ (light/dark) photoperiod, and $50-60 \%$ relative humidity using potato foliage at the vegetative growth or young tuber stages to assure sufficient nutrition. At this feeding protocol, the larvae progressed the first-, second-, penultimate-, and finalinstar stages with approximate periods of 2, 2, 2, and 4 days, respectively. On reaching full size, the final larval instars stopped feeding, dropped to the ground, burrowed to the soil, and entered the prepupal stage. The prepupae spent $\sim 3$ days to pupate. The pupae lasted about 5 days and the adults emerged.

All solvents used for UPLC-Q-TOF-MS analysis were of analytical or HPLC grade and purchased from Sigma-Aldrich Co., Ltd. (Shanghai, China).

The detailed procedure of sample preparation for UPLC-QTOF-MS was as follows: ten (5 males and 5 females) 2-day-old fourth-instar larvae, ten 4-day-old pupae, and ten 5-day-old adults were collected as a replicate. The collection continued for three consecutive generations to generate three biologically independent replicates. The specimens were individually weighed and then crushed in a pre-chilled mortar using a pellet pestle. Each sample was lyophilized, added with $1 \mathrm{ml}$ of methanol/water (7:3, v/v), vortexed, and sonicated twice on ice for $30 \mathrm{~min}$. Then the metabolite was incubated at $-20^{\circ} \mathrm{C}$ for $1 \mathrm{~h}$ and centrifuged at $13,000 \mathrm{rpm}$ at $4^{\circ} \mathrm{C}$ for $15 \mathrm{~min}$. The supernatant was lyophilized and stored at $-80^{\circ} \mathrm{C}$.

\section{Analysis for Operational Taxonomic Units Data From High-Throughput Sequencing of 16S rRNA Genes}

Functional prediction was carried out based on the operational taxonomic units (OTU) data downloaded from the NCBI Sequence Read Archive (SRA) database (Accession Number: PRJNA613266) (Kang et al., 2021). The OTU data were obtained by high-throughput sequencing of $16 \mathrm{~S}$ rRNA genes from three biologically independent collections from three consecutive generations. Each collection included ten 2-day-old fourth-instar larvae, ten 4-day-old pupae, and ten 5-day-old adults with the sex 
ratio of 1:1 (5 males and 5 females). The PICRUSt algorithm was used to infer the functions of the bacterial communities through the Kyoto Encyclopedia of Genes and Genomes (KEGG) database (Kanehisa et al., 2012).

\section{Analysis Conditions of UPLC/Q-TOF-MS}

The supernatant was reconstituted with $500 \mu \mathrm{l}$ of methanol, vortexed, and centrifuged at $20,000 \mathrm{rpm}$ at $4^{\circ} \mathrm{C}$ for $20 \mathrm{~min}$. Then the supernatant was filtered through a $0.22 \mu \mathrm{m}$ (nylon) syringe filter and analyzed by UPLC-Q-TOF-MS system (Waters). Each sample was analyzed six times (i.e., $2 \mu \mathrm{l}$ aliquot of each sample was injected six times).

Since the retention times or even elution order in analytical system may vary during the UPLC-Q-TOF-MS analysis, it is necessary to monitor the system consistency. In this study, a 60 $\mu \mathrm{l}$ mixture of the control $(30 \mu \mathrm{l})$ and treatment samples $(30 \mu \mathrm{l})$ was used as a quality control (QC) sample for method validation. A QC sample ran four times prior to beginning the whole sample list. Moreover, a QC sample was run every six samples during the analytical run.

The analysis was performed on an HSS T3 column $(2.1 \times$ $100 \mathrm{~mm}, 1.8 \mu \mathrm{m}$; Waters Acquity), using an ACQUITY UPLC IClass PLUS System (Waters) coupled with a Xevo ${ }^{\circledR}$ G2-XS QT of High-Definition Mass Spectrometer (Waters). Mobile phase A was water and mobile phase B was $0.1 \%$ formic acid acetonitrile solution. The gradient elution procedure was set as follows: 0$1 \mathrm{~min}, 0-2 \%$ B; 1-2 min, 2-25\% B; 2-4 min, 25-60\% B; 4-7.5 min, $60-90 \%$ B; 7.5-9.5 min, 90-99\% B; 9.5-12.5 min, 99\% B; $12.5-$ $13 \mathrm{~min}, 99-2 \% \mathrm{~B}$; and $13-16 \mathrm{~min}, 2 \% \mathrm{~B}$. The flow rate was $0.4 \mathrm{ml} / \mathrm{min}$, and the column temperatures were held constant at $45^{\circ} \mathrm{C}$.

Each sample was detected by positive and negative ion modes using an electrospray ionization mass spectrometer (ESI-MS). The product ion scan was acquired using the first- and secondlevel mass spectrometry data acquisition method based on the Photodiode Array (PDA) detector.

The ESI conditions were as follows: nitrogen was used as cone gas and desolvation gas at a flow rate of 50 and $800 \mathrm{l} / \mathrm{h}$, respectively. The source temperature was $120^{\circ} \mathrm{C}$ and desolvation gas temperature was $450^{\circ} \mathrm{C}$. Quality scanning range was set $\mathrm{m} / \mathrm{z}$ $50-1,200$. In positive ion mode, capillary, cone, and extraction cone voltages were $3.0 \mathrm{kV}, 40 \mathrm{~V}$, and $5.0 \mathrm{~V}$, respectively. In negative ion mode, capillary, cone, and extraction cone voltages were $2.0 \mathrm{kV}, 40 \mathrm{~V}$, and $5.0 \mathrm{~V}$, respectively. MS data were acquired in full-scan mode from 100 to 1,000 Da.

\section{Data Processing and Statistical Analysis of UPLC/Q-TOF-MS Data}

The raw data detected by UPLC-Q-TOF-MS were loaded on the commercial metabolites database Progenesis QI (Waters Corporation, Milford, USA) for peak detection, alignment, and normalization, as well as the main information, such as the mass, retention time, and intensity of the peaks in each chromatogram. The metabolites were identified by comparing their retention times, $m / z$ values, and MS fragmentation patterns with those of commercial standard compounds. Fragmentation patterns collected in online databases, such as MycompoundID (http:// www.mycompoundid.org), MassBank (http://www.massbank. jp), ChemSpider database (www.chemspider.com), and METLIN (http://metlin.scripps.edu) were also considered, especially when no authentic standard compounds were available.

Before multidimensional statistical analysis, the data were processed: the missing values of the original data $>50 \%$ were excluded. The processed data were then imported into SIMCA-P14.1 software (Umetrics, Umea, Sweden) for pattern recognition, and Pareto scaling was used to preprocess the data for principal component analysis (PCA) and orthogonal PLSDA analysis (OPLS-DA). According to the Variable Importance for the Projection (VIP) obtained by the OPLS-DA model and Max Fold Change (MFC) from Progenesis QI, the influence intensity and explanatory ability of each metabolite on the classification and discrimination of each group of samples were evaluated, and the biologically significant differential metabolites were mined. The larger the VIP and MFC values, the greater the contribution of the metabolite in the differentiation of the sample, and the variable with VIP $>1$ and MFC $>2$ was generally considered to have a significant difference. In the experiment, based on the screening criteria of VIP $>1$ and MFC $>2$, the substances between the groups were initially screened. Next, the univariate statistical analysis was used to verify whether the selected metabolites had significant differences. Ions meeting VIP $>1, \mathrm{MFC}>2$, and $0.05<p<0.1$ were considered differential metabolites; VIP $>1, \mathrm{MFC}>2$, and $p<0.05$ were regarded as significantly different metabolites.

To highlight differential biomarker role, the resulting significant differential metabolites were analyzed in KEGG (http://www.kegg.jp) to resolve the topological trait of metabolic pathways.

\section{Antibiotics Exposure and Examination of Bacteria in Resultant Beetles}

The same method was used to expose the larvae to an antibiotic mixture (Löfmark et al., 2010; Xia et al., 2020). Briefly, a mixture containing three antibiotics $(1 \mathrm{mg} / \mathrm{ml}$ ciprofloxacin, $1 \mathrm{mg} / \mathrm{ml}$ levofloxacin, and $2 \mathrm{mg} / \mathrm{ml}$ metronidazole) in $1 \%$ Tween 20 aqueous solution was used to immerse potato foliage. Tween 20 in sterile water (1\%) was set as the control group. Ten newly ecdysed fourth-instar larvae were confined in a Petri dish $(9 \mathrm{~cm}$ diameter and $1.5 \mathrm{~cm}$ height) containing five treated leaves. The larvae were allowed to feed on the treated leaves until they reached the prepupal stage. The foliage was replaced with freshly treated ones each day.

The total microbial DNAs were individually extracted from 4-day-old pupae having fed on the antibiotic mixture or control solution as larvae, using E.Z.N.A. ${ }^{\circledR}$ Tissue DNA Kit (Omega). The removal of bacteria was examined using a pair of universal primers, the forward primer 5'-TCCTACGGGAGGCAGCAGT- $3^{\prime}$ and reverse primer 5'-GGACTACCAGGGTATCTATCCTGTT-3', of 16S rDNA from the Domain Bacteria (Nadkarni et al., 2002; Silkie and Nelson, 2009). Quantitative DNA measurements were performed by real-time quantitative reverse transcription PCR (qRT-PCR) in technical triplicate. Relative expression level of $16 \mathrm{~S}$ rDNA was 
calculated by the $2^{-\Delta \Delta C T}$ method, using the geometric mean of two internal control genes (LdRP4 and LdARF1) (Shi et al., 2013) for normalization.

To further examine the removal of bacteria, the dissected guts from newly emerged adults in control and treatment groups were homogenized. The supernatants separated by centrifugation at $500 \mathrm{~g}$ were spread on a plate culture medium (Luria-Bertani) after diluting for 10 times. The dishes were inoculated at $30^{\circ} \mathrm{C}$ for $17 \mathrm{~h}$ and then the bacterial spots were detected.

The metabolites in control and bacteria-removed pupae were tested using UPLC-Q-TOF-MS system and the data were analyzed using the method described above.

\section{Statistical Analysis}

Using SPSS for Windows (Chicago, IL, USA), one-way analysis of variance (ANOVA) with a Tukey-Kramer test, or Student's $t$ test was performed to determine significant difference between average values $( \pm \mathrm{SD})$. Results were considered statistically significant when $p<0.05$.

\section{RESULTS}

\section{Functional Analysis of Bacteria}

The OTUs of bacteria obtained by PCR amplification and sequencing of culture-independent 16S rRNA (Kang et al., 2021) were analyzed using KEGG enrichment analysis. At KEGG level 1, metabolism was the most active one, especially in the pupa group (Figure 1A). The highly expressed genes associated with the catabolism of aromatic compounds (at KEGG level 3) were observed in the pupa group, followed by those in the larvae, and the levels were lower in the adult collection. One-way ANOVA revealed that a statistical significant difference was present between pupa and adult groups $(p<0.05)$ (Figure 1B).

Correspondingly, the actively expressed genes (at KEGG level 3 ) involved in the degradation of benzoate, aminobenzoate, styrene, toluene, xylene, nitrotoluene, ethylbenzene, bisphenol, fluorobenzoate, naphthalene, dioxin, steroid, polycyclic aromatic hydrocarbon, caprolactam, atrazine, furfural, chloroalkane, chloroalkene, chlorocyclohexane, and chlorobenzene were significantly richer in the pupa group than those in the larva and adult collections (Figure 1C).

\section{Potential Biomarkers of Aromatic Compounds}

To obtain comprehensive information on the metabolome, small molecule metabolites in the larval, pupal, and adult samples were analyzed by UPLC-Q-TOF-MS. A preliminary analysis of all samples was performed by PCA. The larva and adult groups were biased compared with the pupa collection in both positive and negative ion maps, whereas the larva and adult groups were overlapped (Figures 2A,B).

By OPLS-DA model, 43 potential aromatic biomarkers were screened in positive ion mode. Out of them, a subset of 39 was greater in the pupal specimen compared with those in the larval sample, while another subset of 40 was more in the pupal specimen than those in the adult group (Table 1). A total of 11 potential aromatic biomarkers were obtained in negative ion mode. Among them, subsets of 9 and 8 were greater in the pupal sample than those in the larva and adult groups, respectively (Table 2).

\section{Active Pathway in Pupae}

Biological pathway analysis revealed that five active pathways were involved in the metabolism of aromatics in pupae compared with those in the larva/adult groups. These ways contained the catabolism of styrene, naphthalene, fluorobenzoate, aminobenzoate, and benzoate, respectively (Figures 2C,D).

\section{Enrichment of Xenobiotics in Aposymbiontic Pupa}

Feeding a mixture of three antibiotics by the fourth-instar larvae almost completely removed culturable aerobic bacteria (Figure 3B vs. Figure 3A), and all other bacteria (Figure 3C) in the resultant pupae. In the PCA graphs, the aposymbiontic groups were biased compared with the control groups in both positive and negative ion maps (Figures 3D,E).

In both positive and negative ion modes, the differences in xenobiotic biomarkers between control and aposymbiontic pupal samples included a great number of aromatic compounds (Tables 3, 4).

Metabolic pathway analysis revealed that a total of seven active pathways were enriched in either control or aposymbiontic pupal samples (Figures 3F,G). These ways were involved in the degradation of styrene, naphthalene, benzoate, fluorobenzoate, aminobenzoate, xylene, and dioxin.

\section{Metabolism Pathways}

Identified metabolites and biological pathways were imported into the KEGG (http://www.kegg.jp/) to find interactions. Among networks for catabolism of aromatics, benzoates, 4-methoxybenzoate, vanillin, and benzamide were finally transferred into succinyl-CoA and acetyl-CoA, which were completely degraded to $\mathrm{CO}_{2}$ through tricarboxylic acid cycle (Figures 4A,B,E). Fluorobenzoates, for instance, 3fluorobenzoate and 4-fluorobenzoate, were finally converted to 2-fluoro-cis, cis-muconate, 3-fluoro-cis, cis-muconate, and 4-fluoromuconolactone (Figure 4F). Styrene was oxidized to phenylacetic acid (Figure 4D). In contrast, the degradation of (2naphthyl)methanol (naphthalene) was only partially annotated by KEGG (Figure 4C).

Comparison of metabolite enrichment between control and aposymbiontic pupal samples revealed that 4-methoxybenzoate (Figure 4A), vanillin (Figure 4B), (2-naphthyl)methanol (Figure 4C), styrene oxide, and phenylacetaldehyde (Figure 4D) were higher in the control group. These findings indicated that the soil aromatic precursors could be actively converted into these compounds by pupa bacteria. Conversely, benzoate, styrene, and 3,4-dihydroxybenzoate were accumulated in the aposymbiontic pupal group (Figures 4A,B,E). These results suggested that pupa bacteria were associated with the catabolism of the three chemicals. In contrast, the contents of 3-fluorocatechol and 4-fluorocatechol in control group were similar to those in the aposymbiontic pupal group (Figure 4F). These data implied that pupae may metabolize fluorobenzoates. 
A

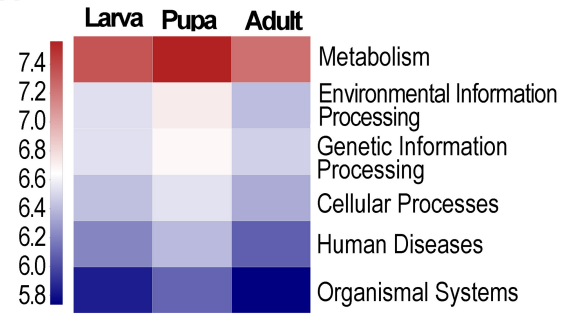

B Degrading aromatic compounds

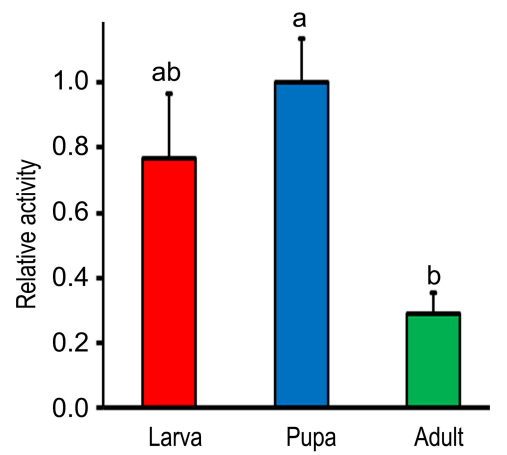

C

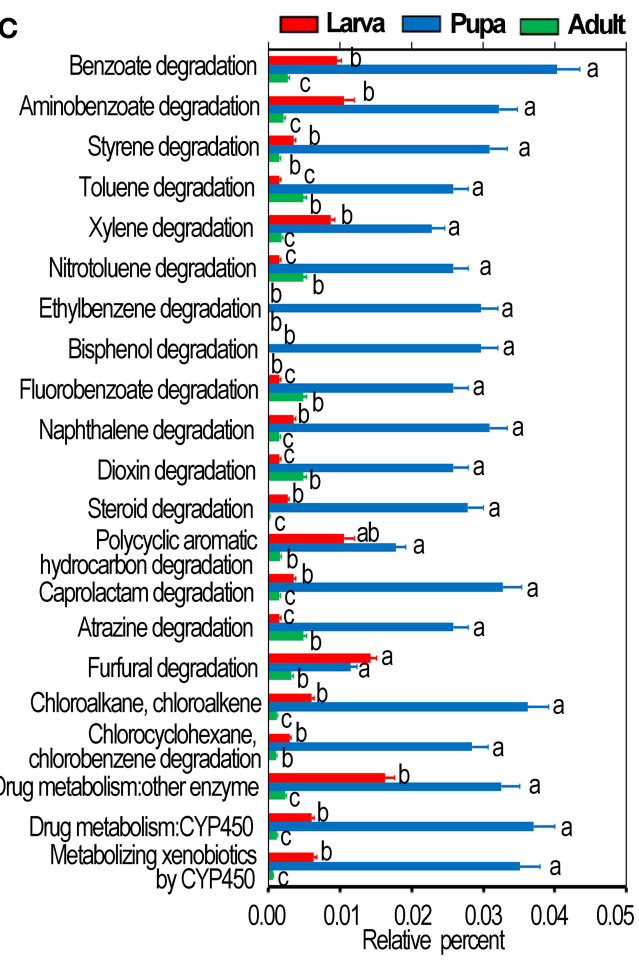

FIGURE 1 | Kyoto Encyclopedia of Genes and Genomes (KEGG) analysis of bacterial flora in Leptinotarsa decemlineata. (A) Shows whole metabolism activities at KEGG level 1. The relative activities in (B) are the ratios of relative percentages in larvae and adults relative to that in the pupae, which is set as 1. (C) Displays the relative activity (percentage) of individual aromatics in the larvae, pupae, and adults at the third KEGG level. The columns represent averages with vertical lines indicating SE. Different letters indicate a significant difference at $p$-value $<0.05$ using analysis of variance with the Tukey-Kramer test.

\section{DISCUSSION}

To the best of our knowledge, the stage-dependent alterations in the biodegradation of aromatics by the stage-specific bacteria flora in insects have not been well-explored. In the present study, PCA analysis of UPLC-Q-TOF-MS data revealed that the larva and adult groups were biased compared with the pupa collection in both positive and negative ion maps (Figure 2). The shift indicates that the metabolites in the pupa group differ from those in the larva and adult groups. In accordance with the indication, the habitat in which the larvae and adults live is different from that of pupae, with the larva and adult settling on potato plants and the pupa in soil (Meng et al., 2019).

\section{The Pupae Mainly Rely on Bacteria to Biodegrade Aromatics}

Incorporation of plant residues and organic fertilizers into soil brings about some secondary chemicals, e.g., alkaloids, terpenoids, cardenolides, glucosinolates, and oxalates (Zhang et al., 2020), and their metabolites such as monoaromatics (e.g., BTEX and phenol) and polycyclic aromatics (e.g., naphthalene, dioxin, and steroids) (Chen et al., 2017; Steinmetz et al., 2019). These compounds often exert deleterious effects to soildwelling pupae when accumulated to high concentrations within the bodies. Insects are hypothesized to specify their microbial community compositions to degrade these substances to avoid intoxication, if suitable ecological conditions are satisfied (Zhang et al., 2020). However, the experimental evidence to support the hypothesis is very limited.

In the present study, we analyzed the bacterial OTU data (Kang et al., 2021) by KEGG and found that a subset of bacterial genes was abundantly expressed at the pupal stage. These genes were associated with the catabolism of aromatics (Figure 1). Consistently, metabolomic analysis displayed that dozens of monoaromatics, polycyclic aromatics, and steroids were richer in the pupal sample than those in the larval and adult specimens (Tables 1, 2).

According to the identified metabolites and biological pathways, a total of seven active ways were enriched. These active pathways are mainly involved in the degradation of various benzoates and their precursors, which include benzoate, 4methoxybenzoate, 3-fluorobenzoate, 4-fluorobenzoate, vanillin (aminobenzoate), and benzamide (Figure 4). Most of these benzoates were finally transferred into succinyl-CoA and acetyl-CoA, which were completely degraded to $\mathrm{CO}_{2}$ through tricarboxylic acid cycle. Similarly, Dechloromonas sp. strains RCB and JJ can completely break down aromatic compounds into $\mathrm{CO}_{2}$, coupled with the reduction of nitrate (Coates et al., 2001). Dechloromonas belongs to proteobacteria. Among the 18 pupaspecific genera identified (Kang et al., 2021), Escherichia_Shigella, 


\section{A Positive ion}

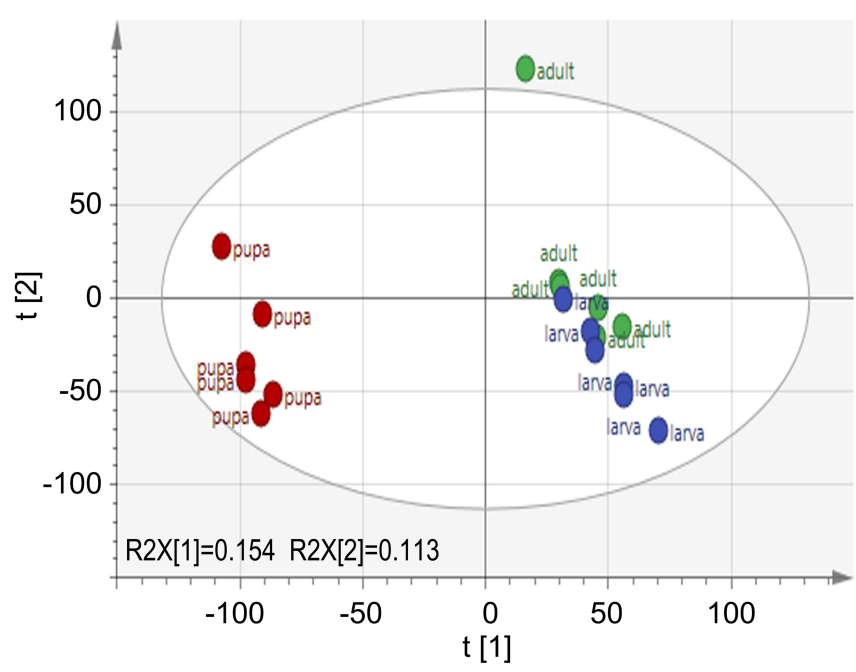

B Negative ion

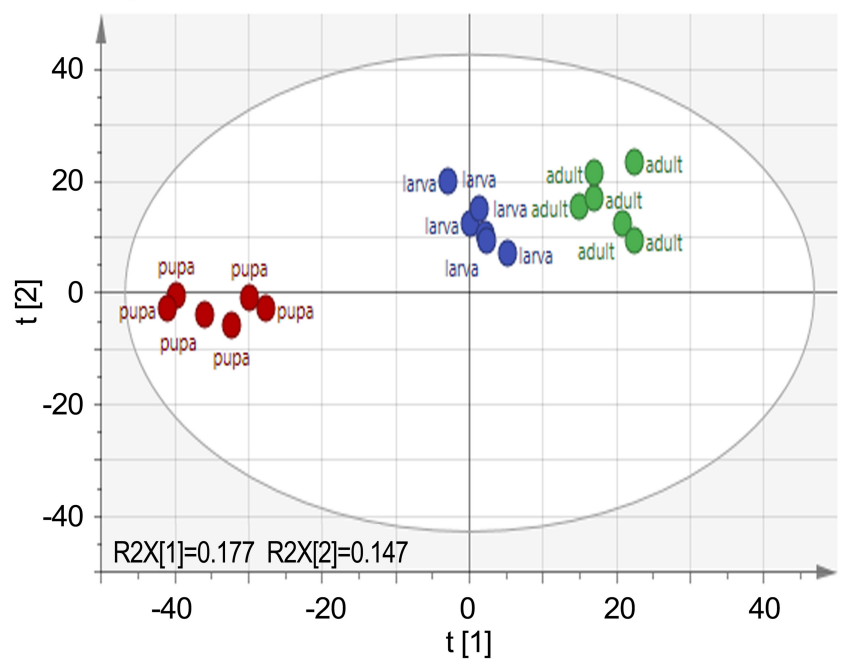

C Pupa v.s. larva

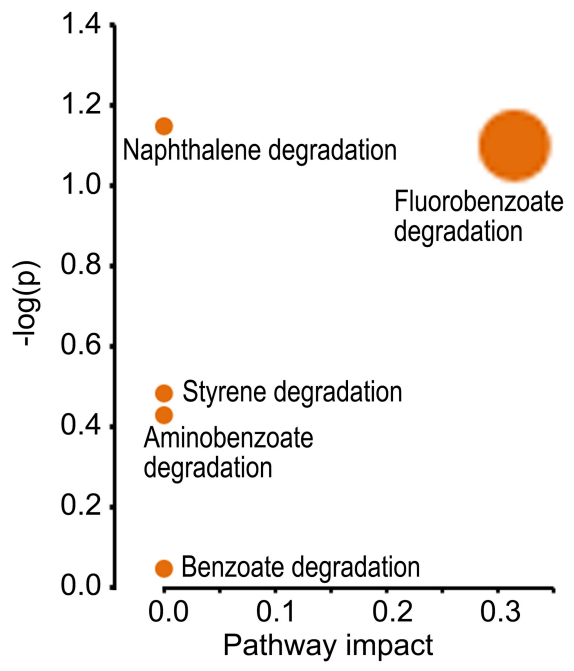

D Pupa v.s. adult

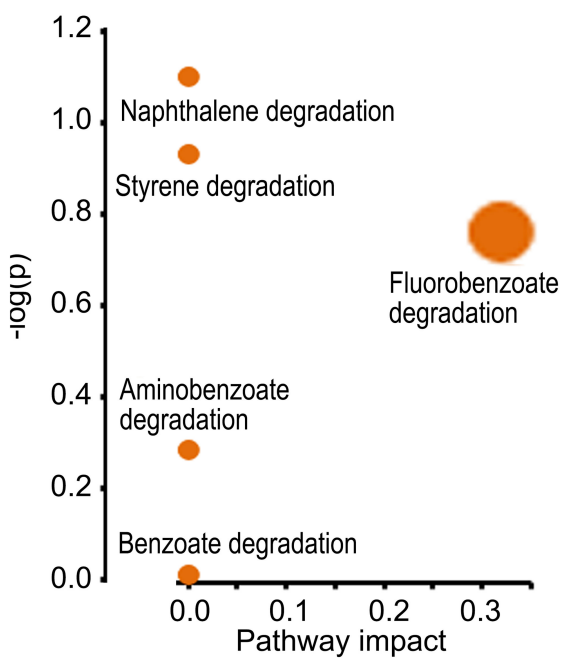

FIGURE 2 | Grouping comparison of metabolomics in larvae, pupae, and adults in L. decemlineata. The ultra-performance liquid chromatography-quadrupole-time of flight mass spectrometry (UPLC-Q-TOF-MS) data were analyzed by principal component analysis (PCA). Positive and negative ion mode PCA score maps (A,B) and pathway enrichment analyses (C,D) of larva, pupa, and adult groups are shown. Different colors in the $(\mathbf{A}, \mathbf{B})$ represented different groups and each point represented a sample.

Acinetobacter, Pseudomonas, Lysobacter, and Stenotrophomonas are also proteobacteria. One or several genera listed above may be responsible for the catabolism of the benzoates in $L$. decemlineata pupae.

Specifically, we uncovered that pupa bacteria were involved in the aerobic degradation of styrene to phenylacetic acid in $L$. decemlineata (Figure 4). In agreement with our result, the way in other documented results involves epoxidation of the vinyl side chain, followed by isomerization of the epoxy styrene to form phenylacetaldehyde. This compound is subsequently oxidized to phenylacetic acid through the action of either an $\mathrm{NAD}^{+}$- or phenazine methosulfate-dependent dehydrogenase (O'Connor and Dobson, 1996; O'Leary et al., 2002). Conversion of styrene to phenylacetic acid has been documented in various bacterial strains; examples include Pseudomonas putida CA-3 (O'Connor et al., 1995), P. fuorescens ST (Marconi et al., 1996), Pseudomonas sp. VLB120 (Panke et al., 1999), Pseudomonas sp. Y2 (Utkin et al., 1991; Velasco et al., 1998), Xanthobacter sp. 124X (Hartmans et al., 1989), and Xanthobacter sp. S5 (Hartmans et al., 1990). Pseudomonas is among the 18 pupa-specific genera identified recently (Kang et al., 2021), and the genus may be responsible for the catabolism of styrene in L. decemlineata pupae.

At present, polycyclic aromatic hydrocarbons produced by all vertebrates as well as some invertebrates have been considered 
TABLE 1 | Differential xenobiotics with higher levels in pupae v.s. larvae/adults in positive ion mode.

\begin{tabular}{|c|c|c|c|c|c|c|c|}
\hline KEGG CID & Metabolites & $P / L$ & P/A & VIP(P/L) & $P(P / L)$ & $\operatorname{VIP}(P / A)$ & $P(P / A)$ \\
\hline $\mathrm{C} 00755$ & Vanillin & $\uparrow$ & $\uparrow$ & 2.2 & $<0.01$ & 2.1 & $<0.01$ \\
\hline C02519 & 4-Methoxybenzoate & $\uparrow$ & $\uparrow$ & 2.2 & $<0.01$ & 2.1 & $<0.01$ \\
\hline C16472 & 3-Fluorocatechol & $\uparrow$ & $\uparrow$ & 4.4 & $<0.01$ & 5.2 & $<0.01$ \\
\hline C16473 & 4-Fluorocatechol & $\uparrow$ & $\uparrow$ & 4.4 & $<0.01$ & 5.2 & $<0.01$ \\
\hline C14110 & 4-Hydroxymethylcatechol & $\uparrow$ & $\uparrow$ & 2.2 & $<0.01$ & 2.1 & $<0.01$ \\
\hline C02909 & (2-Naphthyl)methanol & $\uparrow$ & $\uparrow$ & 4.7 & $<0.01$ & 4.8 & $<0.01$ \\
\hline C00601 & Phenylacetaldehyde & $\uparrow$ & $\uparrow$ & 2.4 & $<0.01$ & 2.5 & $<0.01$ \\
\hline C04043 & 3,4-Dihydroxyphenylacetaldehyde & $\uparrow$ & $\uparrow$ & 2.2 & $<0.01$ & 2.1 & $<0.01$ \\
\hline C02505 & 2-Phenylacetamide & & $\uparrow$ & & & 2.0 & $<0.01$ \\
\hline C02083 & Styrene oxide & $\uparrow$ & $\uparrow$ & 2.4 & $<0.01$ & 1.5 & $<0.01$ \\
\hline C05627 & 4-Hydroxystyrene & $\uparrow$ & $\uparrow$ & 2.4 & $<0.01$ & 1.5 & $<0.01$ \\
\hline C02519 & p-Anisic acid & $\uparrow$ & $\uparrow$ & 2.2 & $<0.01$ & 2.1 & $<0.01$ \\
\hline $\mathrm{C} 07113$ & Acetophenone & $\uparrow$ & $\uparrow$ & 2.4 & $<0.01$ & 1.5 & $<0.01$ \\
\hline C03663 & $2^{\prime}, 4^{\prime}$-Dihydroxyacetophenone & $\uparrow$ & $\uparrow$ & 2.2 & $<0.01$ & 2.1 & $<0.01$ \\
\hline C10675 & $3^{\prime}, 4^{\prime}$-Dihydroxyacetophenone & $\uparrow$ & $\uparrow$ & 2.2 & $<0.01$ & 2.1 & $<0.01$ \\
\hline C13635 & 2,4'-Dihydroxyacetophenone & $\uparrow$ & $\uparrow$ & 2.2 & $<0.01$ & 2.1 & $<0.01$ \\
\hline C15513 & Benzyl acetate & & $\uparrow$ & & & 2.0 & $<0.01$ \\
\hline NA & 4-Hydroxy-3-methylbenzoic acid & $\uparrow$ & $\uparrow$ & 2.2 & $<0.01$ & 2.1 & $<0.01$ \\
\hline C10804 & 2-(Hydroxymethyl)benzoic acid & $\uparrow$ & $\uparrow$ & 2.2 & $<0.01$ & 2.1 & $<0.01$ \\
\hline $\mathrm{C} 02181$ & Phenoxyacetic acid & $\uparrow$ & $\uparrow$ & 2.2 & $<0.01$ & 2.1 & $<0.01$ \\
\hline NA & 3-Cresotinic acid & $\uparrow$ & $\uparrow$ & 2.2 & $<0.01$ & 2.1 & $<0.01$ \\
\hline C14103 & 4-Methylsalicylate & $\uparrow$ & $\uparrow$ & 2.2 & $<0.01$ & 2.1 & $<0.01$ \\
\hline C08157 & Testosterone enanthate & $\uparrow$ & $\uparrow$ & 1.2 & $<0.01$ & 1.2 & $<0.01$ \\
\hline NA & Pregnanetriol & $\uparrow$ & $\uparrow$ & 3.7 & $<0.01$ & 3.9 & $<0.01$ \\
\hline C14606 & 11-Hydroxyandrosterone & $\uparrow$ & & 1.1 & 0.0145 & & \\
\hline NA & Saponin $\mathrm{H}$ & $\uparrow$ & $\uparrow$ & 1.5 & $<0.01$ & 1.4 & $<0.01$ \\
\hline NA & Assamsaponin D & $\uparrow$ & $\uparrow$ & 1.5 & $<0.01$ & 1.4 & $<0.01$ \\
\hline NA & 2,3-Dihydrobenzofuran & $\uparrow$ & $\uparrow$ & 2.4 & $<0.01$ & 2.5 & $<0.01$ \\
\hline C11168 & Tetrabenazine & $\uparrow$ & $\uparrow$ & 1.6 & $<0.01$ & 1.5 & $<0.01$ \\
\hline C12508 & Nateglinide & $\uparrow$ & $\uparrow$ & 1.6 & $<0.01$ & 1.5 & $<0.01$ \\
\hline NA & Eremopetasinorol & $\uparrow$ & & 1.7 & $<0.01$ & & \\
\hline NA & Chloropanaxydiol & $\uparrow$ & & 1.0 & $<0.01$ & & \\
\hline
\end{tabular}

P/L, pupal group vs. larval group; P/A, pupal group v.s. adult group; $\uparrow$, upregulation.

one of the most important environmental problems (Xiong et al., 2019; Chiang et al., 2020). In this study, we discovered that the polycyclic aromatic hydrocarbon (2-naphthyl)methanol (naphthalene) can be biodegraded by pupa-specific bacteria in $L$. decemlineata (Figure 4). It has been reported that naphthalene can be catabolized by $P$. fluorescens AH-40 (Mawad et al., 2020), P. putida BS3701 (Pozdnyakova-Filatova et al., 2020), and Stenotrophomonas sp. S1VKR-26 (Patel and Patel, 2020). Naphthalene is converted via salicylate and catechol to the intermediates of tricarboxylic acid cycle in P. putida PpG1 (Yen and Gunsalus, 1982), catalyzed by four key enzymes, namely, naphthalene 1,2-dioxygenase, salicylate hydroxylase, catechol 2,3-dioxygenase, and catechol 1,2-dioxygenase (Izmalkova et al., 2006). In this survey, we determined that 3 - and 4-fluorocatechol, 
TABLE 2 | Differential xenobiotics with higher levels in pupae v.s. larvae/adults in negative ion mode.

\begin{tabular}{|c|c|c|c|c|c|c|c|}
\hline KEGG CID & Metabolites & P/L & P/A & VIP(P/L) & $P(P / L)$ & VIP(P/A) & $P(P / A)$ \\
\hline NA & 4-Hydroxybenzylamine & & $\uparrow$ & & & 1.2 & $<0.01$ \\
\hline C10770 & 5-(8-Pentadecenyl)-1,3-benzenediol & $\uparrow$ & $\uparrow$ & 1.3 & $<0.01$ & 1.9 & 0.016 \\
\hline C03719 & Phenylacetothiohydroximate & $\uparrow$ & $\uparrow$ & 1.8 & $<0.01$ & 1.7 & $<0.01$ \\
\hline C08158 & Testosterone propionate & $\uparrow$ & $\uparrow$ & 5.9 & $<0.01$ & 5.3 & $<0.01$ \\
\hline C14643 & Medrysone & $\uparrow$ & $\uparrow$ & 5.9 & $<0.01$ & 5.3 & $<0.01$ \\
\hline C08185 & Triamcinolone hexacetonide & $\uparrow$ & $\uparrow$ & 1.7 & 0.02 & 1.6 & 0.02 \\
\hline C07119 & Medroxyprogesterone & & $\uparrow$ & & & 5.3 & $<0.01$ \\
\hline C01780 & Aldosterone & $\uparrow$ & & 1.0 & $<0.01$ & & \\
\hline C18039 & Aldosterone hemiacetal & $\uparrow$ & & 1.0 & $<0.01$ & & \\
\hline C19873 & 26-Hydroxycastasterone & & $\uparrow$ & & & 1.7 & $<0.01$ \\
\hline C19326 & p-Anisidine & $\uparrow$ & $\uparrow$ & 1.5 & 0.01 & 1.2 & $<0.01$ \\
\hline C07369 & Prednisolone & $\uparrow$ & & 1.0 & 0.01 & & \\
\hline
\end{tabular}

P/L, pupal group vs. larval group; P/A, pupal group v.s. adult group; $\uparrow$, upregulation.

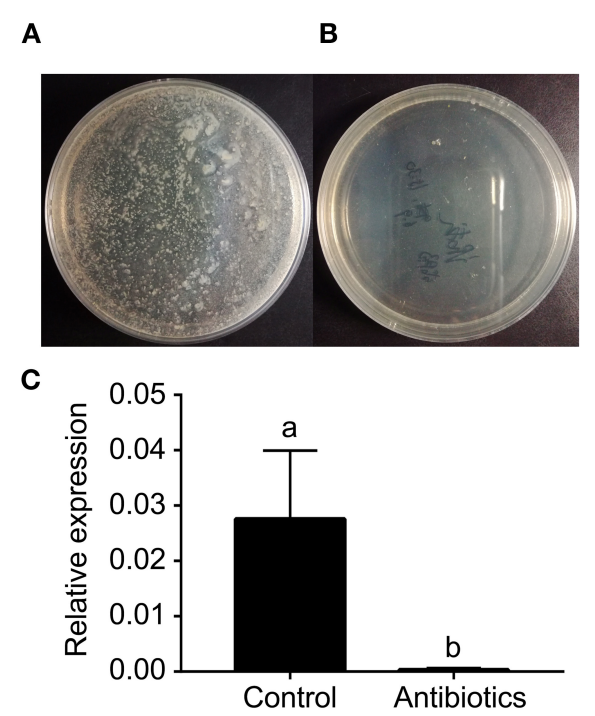

D Positive ion

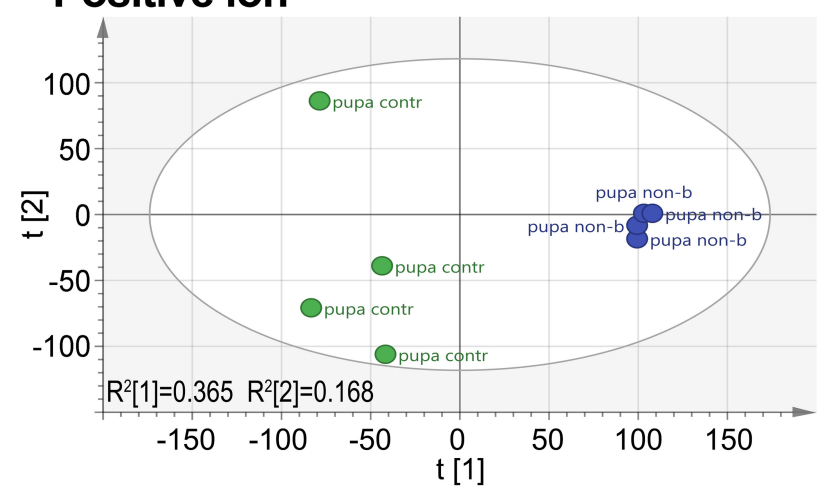

F Enrichment in control

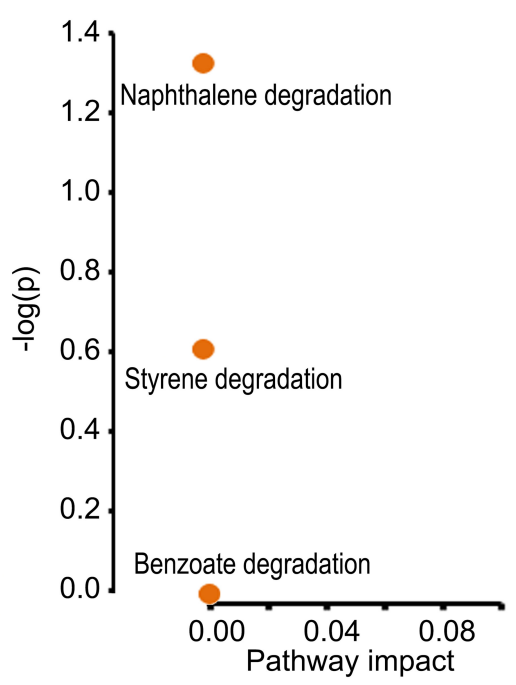

\section{G Enrichment in treatment}

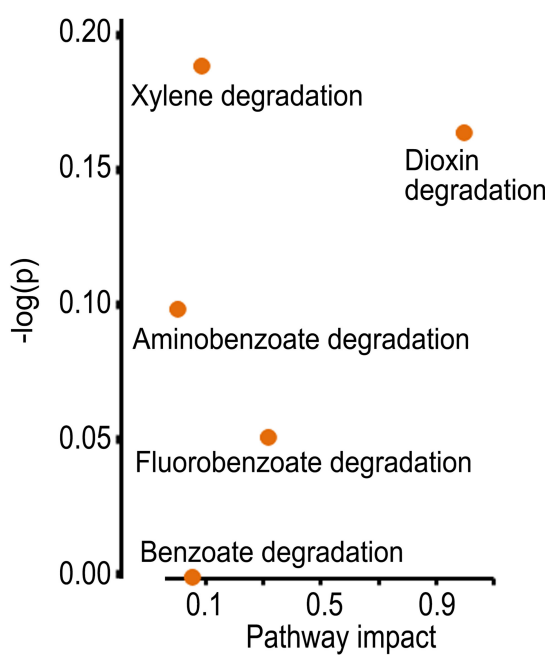

\section{E Negative ion}

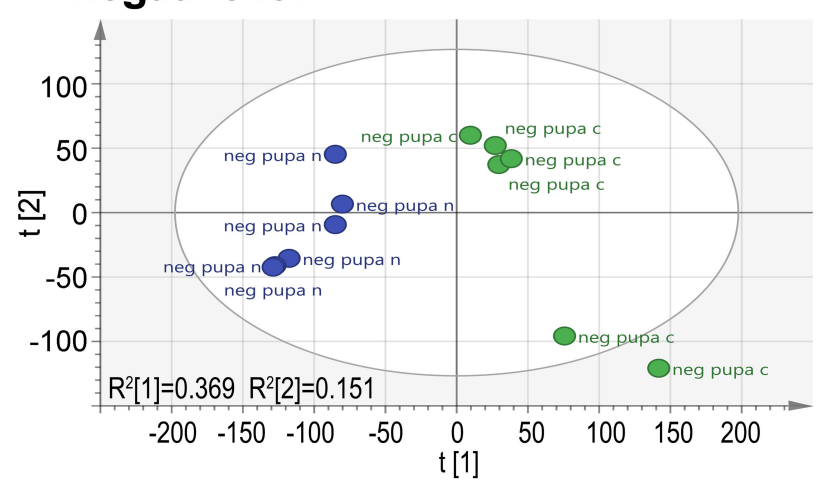

FIGURE 3 | Grouping comparison of metabolomics in control and antibiotic-fed pupae in L. decemlineata. The removal of bacteria was examined by plate culture $(\mathbf{A}, \mathbf{B})$ and qRT-PCR using a pair of universal primers of $16 \mathrm{~S}$ rDNA from the Domain Bacteria (C). Relative expression level of $16 \mathrm{~S}$ rDNA was calculated by the $2^{-} \Delta \Delta \mathrm{CT}$ method. Different letters indicate significant difference at $p$-value $<0.05$ using analysis of variance with the Tukey-Kramer test. The UPLC-Q-TOF-MS resultant data were analyzed by PCA. Positive and negative ion mode PCA score maps (D,E) and pathway enrichment analyses (F,G) in control vs. antibiotic-fed pupal samples are shown. Green and blue colors in the (D,E) represented control and aposymbiontic pupal groups and each point represented a sample. 
TABLE 3 | Differential xenobiotic and other secondary metabolite biomarkers with higher levels in controls than antibiotic-fed pupae in positive ion mode.

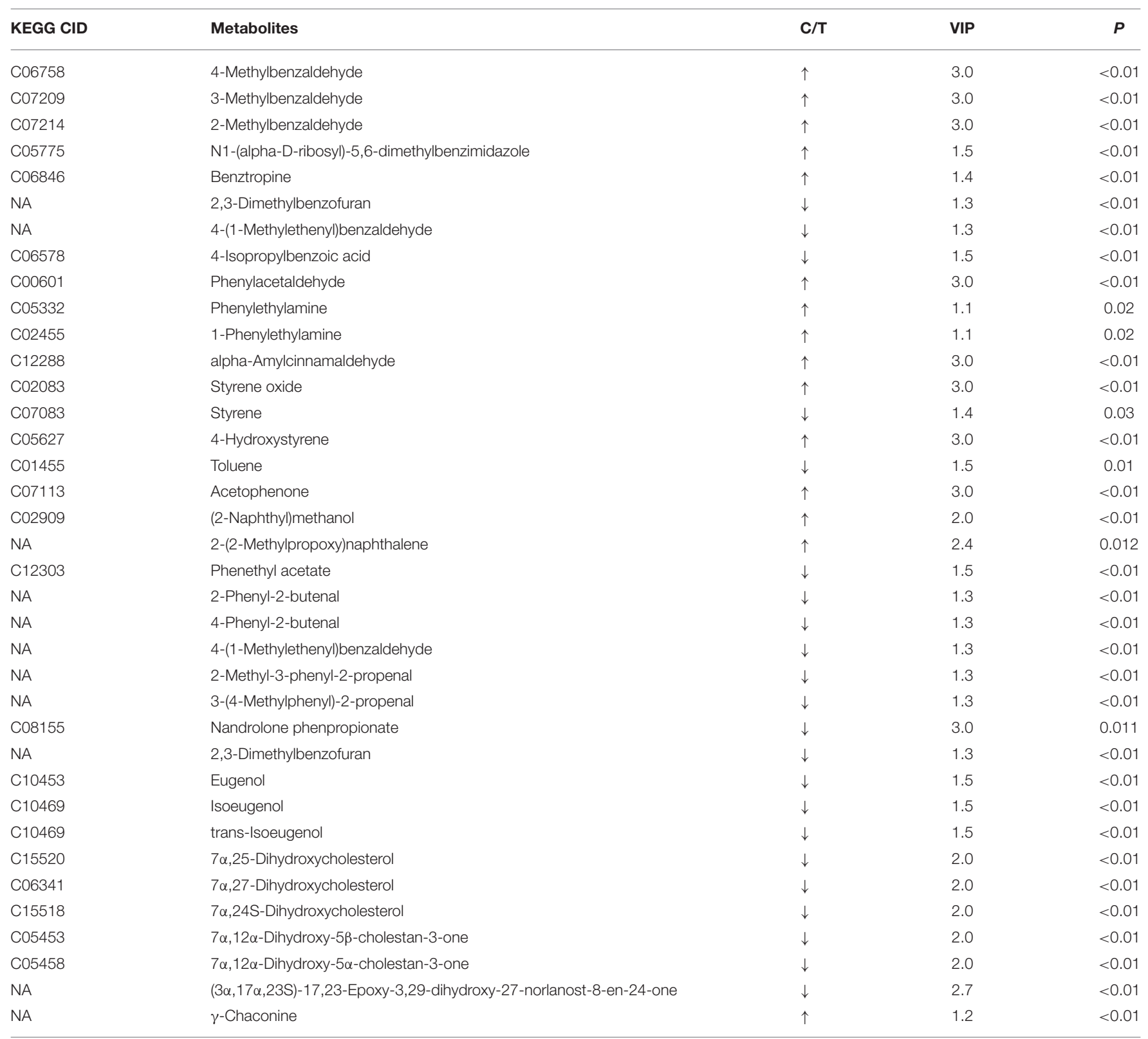

C/T, pupal control group v.s. pupae treated with antibiotics group; $\uparrow$, upregulation; $\downarrow$, downregulation.

4-hydroxymethylcatechol, and 4-methylsalicylate were higher in the pupae than those in the larvae and adults. It appears that the same naphthalene transformation way is present in the L. decemlineata pupae. Consistent with the metabolomic result, our microbiome analysis revealed that both Pseudomonas and Stenotrophomonas are among the 18 pupa-specific genera identified recently (Kang et al., 2021).

Both biogenic (natural) and anthropogenic steroids are frequently detected in soils and aquatic environments in China (Chiang et al., 2020). For example, oestrogens, androgens, progestogens, glucocorticoids, and mineralocorticoids are detected in the surface water of urban rivers in Beijing (Chang et al., 2009). Bacteria are responsible for mineralizing polycyclic aromatic hydrocarbons from the biosphere (Chiang et al., 2020). Among 18 pupa-specific bacterial genera (Kang et al., 2021), Nocardia (Coombe et al., 1966), Rhodococcus (Fernandez de las Heras et al., 2009; Li et al., 2018), and Stenotrophomonas (Juhasz et al., 2000; Tachibana et al., 2003; Guan et al., 2019; Xiong et al., 2019) have been documented to degrade steroids from other environments. Whether the three bacterial genera in $L$. decemlineata can break down steroids deserves further research.

Saponins are a class of secondary plant metabolites which includes triterpenoids, steroids, and steroidal alkaloids glycosylated with one or more sugar chains. They are produced by many plant species (Zhang et al., 2020). Saponins provoke molting defects in, and exert deleterious effects on insects (De 
TABLE 4 | Differential xenobiotics with higher levels in controls than antibiotic-fed pupae in negative ion mode.

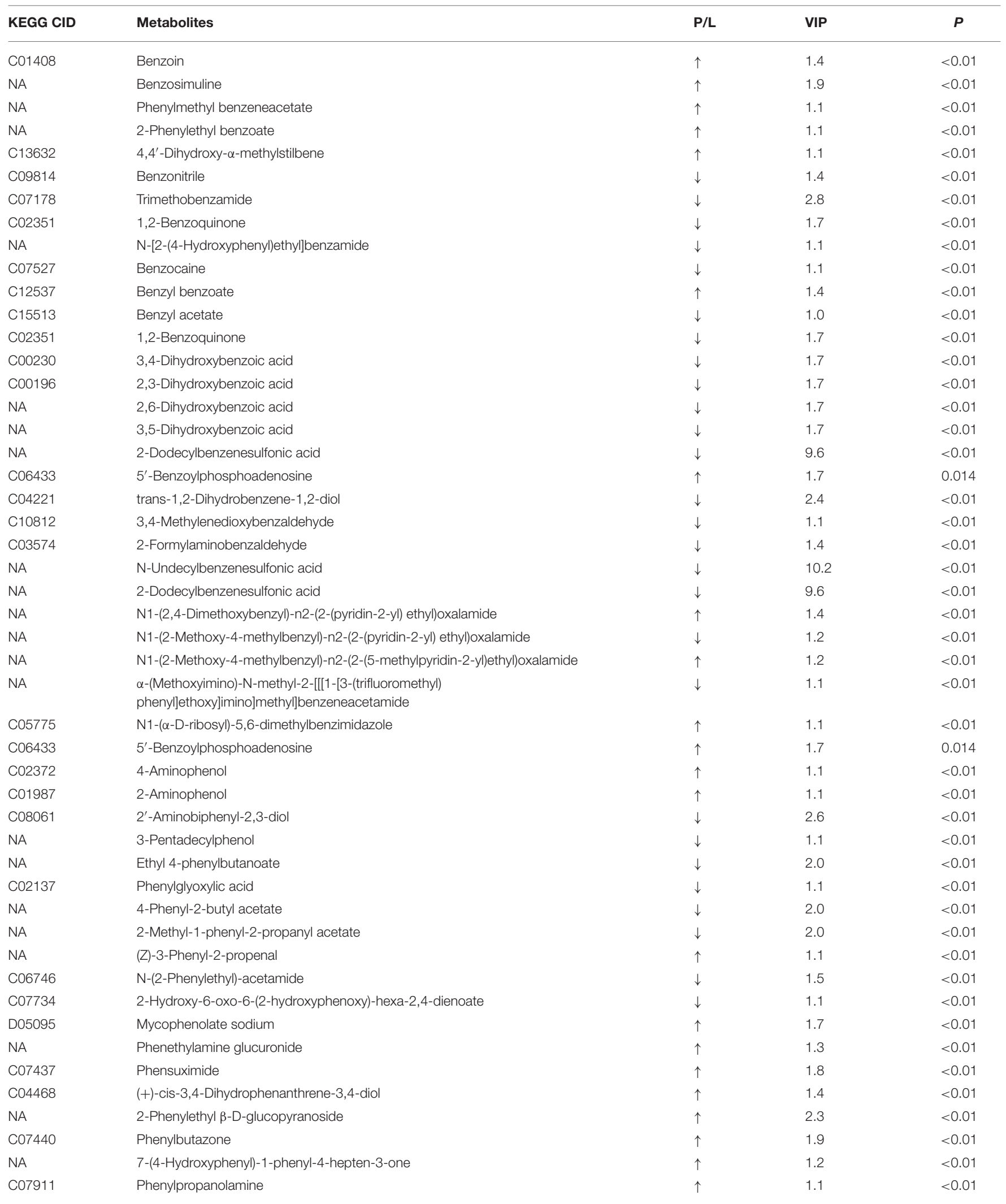


TABLE 4 | Continued

\begin{tabular}{|c|c|c|c|c|}
\hline KEGG CID & Metabolites & $P / L$ & VIP & $\boldsymbol{P}$ \\
\hline NA & (Z)-3-Phenyl-2-propenal & $\uparrow$ & 1.1 & $<0.01$ \\
\hline NA & Phenylmethyl benzeneacetate & $\uparrow$ & 1.1 & $<0.01$ \\
\hline C03719 & Phenylacetothiohydroximate & $\uparrow$ & 5.3 & $<0.01$ \\
\hline NA & 2-Phenylethyl benzoate & $\uparrow$ & 1.1 & $<0.01$ \\
\hline NA & 1-(5-Acetyl-2-hydroxyphenyl)-3-methyl-1-butanone & $\uparrow$ & 1.4 & $<0.01$ \\
\hline C02633 & 20-Hydroxyecdysone & $\uparrow$ & 1.4 & $<0.01$ \\
\hline C02513 & 3-Dehydroecdysone & $\uparrow$ & 1.1 & $<0.01$ \\
\hline NA & 16-Oxoestrone & $\uparrow$ & 2.1 & $<0.01$ \\
\hline C00468 & Estrone & $\uparrow$ & 1.9 & $<0.01$ \\
\hline C02537 & $17 \alpha$-Estradiol & $\uparrow$ & 4.4 & $<0.01$ \\
\hline C05295 & 19-Oxotestosterone & $\uparrow$ & 4.4 & $<0.01$ \\
\hline NA & Zapotin & $\uparrow$ & 1.7 & $<0.01$ \\
\hline C05485 & 21-Hydroxypregnenolone & $\downarrow$ & 3.8 & $<0.01$ \\
\hline C18038 & $7 \alpha$-Hydroxypregnenolone & $\downarrow$ & 3.8 & $<0.01$ \\
\hline C06390 & $16 \alpha$-Hydroxypregnenolone & $\downarrow$ & 3.8 & $<0.01$ \\
\hline C05138 & $17 \alpha$-Hydroxypregnenolone & $\downarrow$ & 3.8 & $<0.01$ \\
\hline C04518 & (20S)-17,20-Dihydroxypregn-4-en-3-one & $\downarrow$ & 3.8 & $<0.01$ \\
\hline C18040 & $5 \alpha$-Dihydrodeoxycorticosterone & $\downarrow$ & 3.8 & $<0.01$ \\
\hline
\end{tabular}

$C / T$, pupal control group v.s. pupae treated with antibiotics group; $\uparrow$, upregulation; $\downarrow$, downregulation.

Geyter et al., 2007; Podolak et al., 2010; Cai et al., 2016; Dolma et al., 2017). Therefore, tea saponin has been widely used as an insecticide in China (Cai et al., 2016). We herein demonstrated that the contents of saponin $\mathrm{H}$ and assamsaponin $\mathrm{D}$ were higher in the pupae compared with those in the larvae and adults in $L$. decemlineata (Figure 4).

Although a considerable amount of saponins is indicated in the soil, L. decemlineata pupae could still develop into adults, indicating that other factors may help $L$. decemlineata resist saponins. Some bacteria, for instance, Acinetobacter calcoaceticus and A. oleivorans, are known to detoxify saponins (Zhang et al., 2020). Moreover, the mixed cultures of Methanobrevibacter spp. and Methanosphaera stadtmanae in the crop of the avian foregut fermenter in Opisthocomus hoazin are able to reduce the hemolytic activity of Quillaja saponins by $80 \%$ within a few hours (García-Amado et al., 2007). Consistent with these results, Acinetobacter is a pupa-specific genus (Kang et al., 2021), and it may be responsible for the metabolism of saponins in the $L$. decemlineata pupae.

\section{Elimination of Aromatics in the Larvae and Adults}

Potato plants contain many aromatics, where some exert noxious effects when accumulated to high concentrations within insect bodies (Gandia-Herrero and Garcia-Carmona, 2013; Kostyn et al., 2020). In this survey, we discovered that only 6 and 7 aromatics were accumulated in the larvae and adults, respectively, in contrast to 42 cumulated aromatics in the pupae (Tables 1, 2). The less accumulation implies the more active elimination of aromatics in the $L$. decemlineata larvae and adults.

Consistently, the microbiota are widely distributed in the larval or/and adult guts (Jing et al., 2020). These gut microbiota are involved in the breakdown of noxious compounds in numerous insect species in Coleoptera (Ceja-Navarro et al., 2015; Berasategui et al., 2017; Zhang et al., 2020), Lepidoptera (Vilanova et al., 2016; Zeng et al., 2020), Diptera (Griffin and Reed, 2020), Hymenoptera (Wu et al., 2020), and Isoptera (Van Dexter and Boopathy, 2019).

In this survey, the bacterial OTU data (Kang et al., 2021) revealed that the catabolism of aromatics was less active in the larvae and adults compared with that in L. decemlineata pupae (Figure 1). Therefore, the bacterial biodegradation of aromatics only partially contributes to the removal of excessive aromatics. Intestinal excretion should be another route to eliminate superfluous aromatics (Rozman, 1985). In fact, insecticides can be excreted by insects, directly or indirectly (modified forms) (Quesada et al., 2020). In L. decemlineata larvae and adults, superfluous aromatics in food may be excreted through the guts directly, or transferred to more hydrophilic forms. Only those potato aromatics absorbed by $L$. decemlineata larvae and adults need to be biodegraded by bacteria. Conversely, the alimentary canal is not well-developed in L. decemlineata pupae and cannot actively remove excessive aromatics. The pupae mainly depend on bacteria to catabolize the noxious substances. 


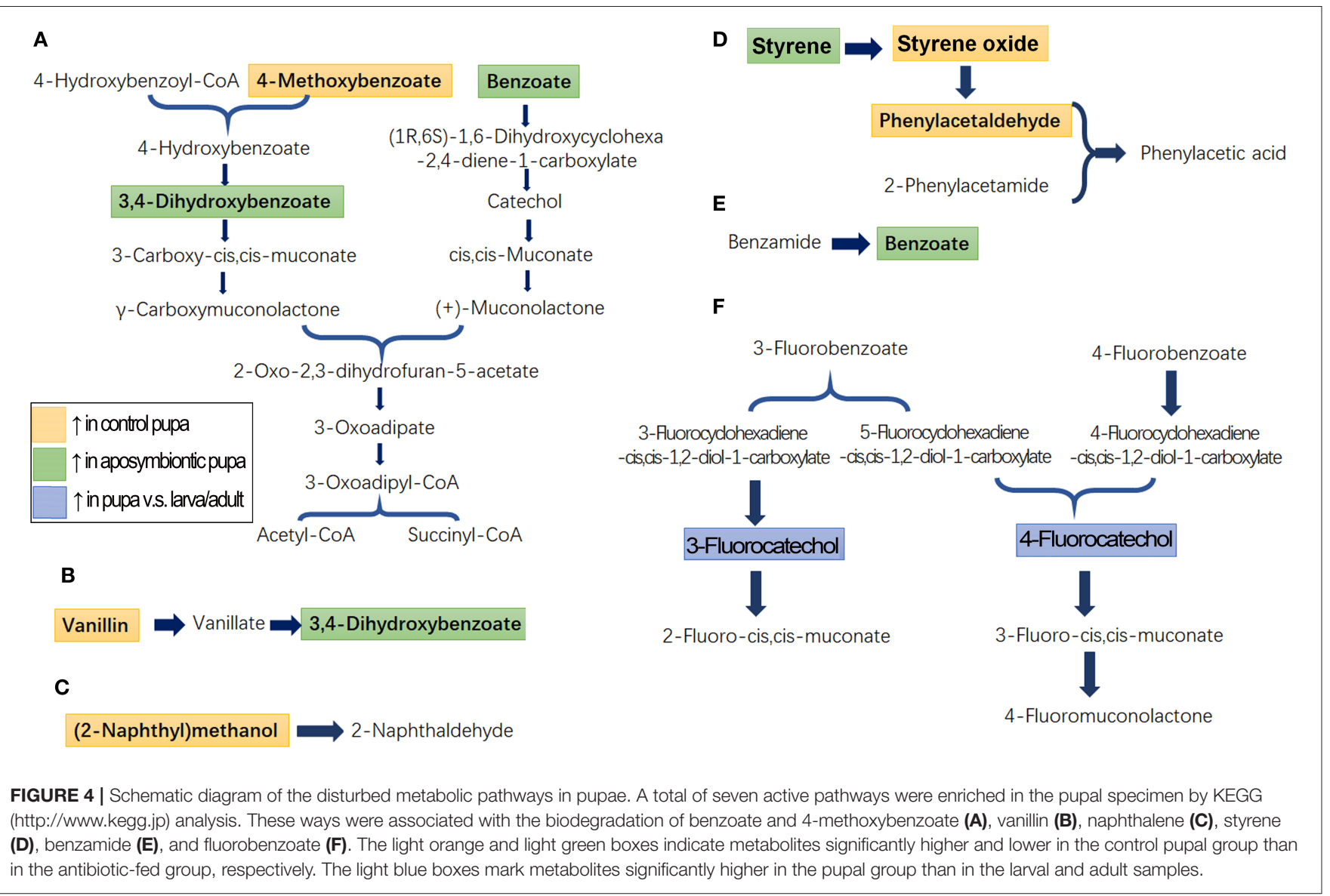

In summary, we uncovered the stage-dependent alterations in bacterial degradation of aromatics in L. decemlineata. The candidate bacterial genera contributing to aromatic catabolism were Nocardia, Rhodococcus, Enterococcus, Acinetobacter, Pseudomonas, and Stenotrophomonas, among others. This study provides new insights into the adaptation of $L$. decemlineata to different environmental niches and offers a better understanding of the relationship between ONS and a shift of bacterial flora. Moreover, since removal of the symbiotic bacteria inhibited the breakdown of superfluous aromatics (this study) and results in a decrease in the emergence rate and adult weight (Kang et al., 2021), disruption of bacterial communities may be a potential strategy to control L. decemlineata.

\section{DATA AVAILABILITY STATEMENT}

The datasets presented in this study can be found in online repositories. The names of the repository/repositories

\section{REFERENCES}

Berasategui, A., Salem, H., Paetz, C., Santoro, M., Gershenzon, J., Kaltenpoth, M., et al. (2017). Gut microbiota of the pine weevil degrades conifer diterpenes and increases insect fitness. Mol. Ecol. 26, 4099-4110. doi: 10.1111/mec.14186 and accession number(s) can be found in the article/supplementary material.

\section{AUTHOR CONTRIBUTIONS}

W-NK, LJ, H-YM, and G-QL conceived the study, and participated in the design of the experiments and the interpretation of the results. W-NK, LJ, and H-YM performed the experiments. W-NK, LJ, and G-QL wrote the first draft of the manuscript. All authors contributed to the article and approved the submitted version.

\section{FUNDING}

This research was supported by the National Natural Science Foundation of China (32072416), and the China Agriculture Research System of MOF and MARA (CARS-09-P22). 
Ceja-Navarro, J. A., Vega, F. E., Karaoz, U., Hao, Z., Jenkins, S., Lim, H. C., et al. (2015). Gut microbiota mediate caffeine detoxification in the primary insect pest of coffee. Nat. Commun. 6:7618. doi: 10.1038/ncomms8618

Chang, H., Wan, Y., and Hu, J. (2009). Determination and source apportionment of five classes of steroid hormones in urban rivers. Environ. Sci. Technol. 43, 7691-7698. doi: 10.1021/es803653j

Chen, B., Teh, B. S., Sun, C., Hu, S., Lu, X., Boland, W., et al. (2016). Biodiversity and activity of the gut microbiota across the life history of the insect herbivore Spodoptera littoralis. Sci. Rep. 6:29505. doi: 10.1038/srep29505

Chen, J., Zhang, L., Jin, Q., Su, C., Zhao, L., Liu, X., et al. (2017). Bioremediation of phenol in soil through using a mobile plant-endophyte system. Chemosphere 182, 194-202. doi: 10.1016/j.chemosphere.2017.05.017

Chiang, Y.-R., Wei, S. T.-S., Wang, P.-H., Wu, P.-H., and Yu, C.-P. (2020). Microbial degradation of steroid sex hormones: implications for environmental and ecological studies. Microb. Biotechnol. 13, 926-949. doi: 10.1111/1751-7915.13504

Coates, J. D., Chakraborty, R., Lack, J. G., O'Connor, S. M., Cole, K. A., Bender, K. S., et al. (2001). Anaerobic benzene oxidation coupled to nitrate reduction in pure culture by two strains of Dechloromonas. Nature 411, 1039-1043. doi: $10.1038 / 35082545$

Coombe, R. G., Tsong, Y. Y., Hamilton, P. B., and Sih, C. J. (1966). Mechanisms of steroid oxidation by microorganisms. X. Oxidative cleavage of estrone. J. Biol. Chem. 241, 1587-1595. doi: 10.1016/S0021-9258(18)96753-0

De Geyter, E., Lambert, E., Geelen, D., and Smagghe, G. (2007). "Novel advances with plant saponins as natural insecticides to control pest insects," in Pest Technology Global Science Books, 96-105.

Dolma, S. K., Sharma, E., Gulati, A., and Reddy, S. G. E. (2017). Insecticidal activities of tea saponin against diamondback moth, Plutella xylostella and aphid, Aphis craccivora. Toxin Rev. 37, 52-55. doi: 10.1080/15569543.2017.1318405

Fernandez de las Heras, L., Garcia Fernandez, E., Maria Navarro Llorens, J., Perera, J., and Drzyzga, O. (2009). Morphological, physiological, and molecular characterization of a newly isolated steroid-degrading actinomycete, identified as Rhodococcus ruber strain Chol-4. Curr. Microbiol. 59, 548-553. doi: 10.1007/s00284-009-9474-Z

Fürstenberg-Hägg, J., Zagrobelny, M., and Bak, S. (2013). Plant defense against insect herbivores. Int. J. Mol. Sci. 14, 10242-10297. doi: 10.3390/ijms140510242

Gandia-Herrero, F., and Garcia-Carmona, F. (2013). Biosynthesis of betalains: yellow and violet plant pigments. Trends Plant Sci. 18, 334-343. doi: 10.1016/j.tplants.2013.01.003

García-Amado, M. A., Michelangeli, F., Gueneau, P., Perez, M. E., and DominguezBello, M. G. (2007). Bacterial detoxification of saponins in the crop of the avian foregut fermenter Opisthocomus hoazin. J. Anim. Feed Sci. 16, 82-85. doi: $10.22358 /$ jafs/74460/2007

Griffin, L. H., and Reed, L. K. (2020). Effect of gut microbiota on $\alpha$-amanitin tolerance in Drosophila tripunctata. Ecol. Evol. 10, 9419-9427. doi: 10.1002/ece 3.6630

Guan, J., Wang, H., Zhu, Y., and Xu, J. (2019). Whole-genome sequencing of S. maltophilia and degradation of fluoranthene. Genomics Appl. Biol. 38, 3037-3045. doi: 10.13417/j.gab.038.003037

Hammer, T. J., and Bowers, M. D. (2015). Gut microbes may facilitate insect herbivory of chemically defended plants. Oecologia 179, 1-14. doi: 10.1007/s00442-015-3327-1

Hanselman, T. A., Graetz, D. A., and Wilkie, A. C. (2003). Manure-borne estrogens as potential environmental contaminants: a review. Environ. Sci. Technol. 37, 5471-5478. doi: 10.1021/es034410+

Hartmans, S., Smits, J. P., van der Werf, M. J., Volkering, F., and de Bont, J. A. (1989). Metabolism of styrene oxide and 2-phenylethanol in the styrenedegrading Xanthobacter strain 124X. Appl. Environ. Microbiol. 55, 2850-2855. doi: 10.1128/aem.55.11.2850-2855.1989

Hartmans, S., van der Werf, M. J., and de Bont, J. A. (1990). Bacterial degradation of styrene involving a novel flavin adenine dinucleotidedependent styrene monooxygenase. Appl. Environ. Microbiol. 56, 1347-1351. doi: 10.1128/aem.56.5.1347-1351.1990

Izmalkova, T. Y., Mavrodi, D. V., Sokolov, S. L., Kosheleva, I. A., Smalla, K., Thomas, C. M., et al. (2006). Molecular classification of IncP-9 naphthalene degradation plasmids. Plasmid 56, 1-10. doi: 10.1016/j.plasmid.2005.12.004
Jing, T. Z., Qi, F. H., and Wang, Z. Y. (2020). Most dominant roles of insect gut bacteria: digestion, detoxification, or essential nutrient provision? Microbiome 8:38. doi: 10.1186/s40168-020-00823-y

Juhasz, A. L., Stanley, G. A., and Britz, M. L. (2000). Microbial degradation and detoxification of high molecular weight polycyclic aromatic hydrocarbons by Stenotrophomonas maltophilia strain VUN 10,003. Lett. Appl. Microbiol. 30, 396-401. doi: 10.1046/j.1472-765x.2000.00733.x

Kanehisa, M., Goto, S., Sato, Y., Furumichi, M., and Tanabe, M. (2012). KEGG for integration and interpretation of largescale molecular data sets. Nucleic Acids Res. 40, 109-114. doi: 10.1093/nar/gkr988

Kang, W.-N., Jin, L., Fu, K.-Y., Guo, W.-C., and Li, G.-Q. (2021). A switch of microbial flora coupled with ontogenetic niche shift in Leptinotarsa decemlineata. Arch. Insect Biochem. Physiol. 106:e21782. doi: 10.1002/arch.21782

Kjaer, J., Olsen, P., Bach, K., Barlebo, H. C., Ingerslev, F., Hansen, M., et al. (2007). Leaching of estrogenic hormones from manure-treated structured soils. Environ. Sci. Technol. 41, 3911-3917. doi: 10.1021/es0627747

Kostyn, K., Boba, A., Kostyn, A., Kozak, B., Starzycki, M., Kulma, A., et al. (2020). Expression of the tyrosine hydroxylase gene from rat leads to oxidative stress in potato plants. Antioxidants 9:717. doi: 10.3390/antiox9080717

Kumar, K., and Goh, K. M. (2000). Crop residues and management practices: effects on soil quality, soil nitrogen dynamics, crop yield, and nitrogen recovery. Adv. Agron. 68, 197-319. doi: 10.1016/S0065-2113(08)60846-9

Lesperance, D. N., and Broderick, N. A. (2020). Microbiomes as modulators of Drosophila melanogaster homeostasis and disease. Curr. Opin. Insect Sci. 39, 84-90. doi: 10.1016/j.cois.2020.03.003

Li, Y., Wang, H. Q., Wu, X. X., and Xu, J. (2018). Study on the trans-membrane transport process of fluoranthene by Rhodococcus sp. BAP-1. China Environ. Sci. 38, 1441-1448. doi: 10.19674/j.cnki.issn1000-6923.2018.0174

Löfmark, S., Edlund, C., and Nord, C. E. (2010). Metronidazole is still the drug of choice for treatment of anaerobic infections. Clin. Infect. Dis. 50, S16-S23. doi: 10.1086/647939

Marconi, A. M., Beltrametti, F., Bestetti, G., Solinas, F., Ruzzi, M., Galli, E., et al. (1996). Cloning and characterisation of styrene catabolism genes from Pseudomonas fuorescens ST. Appl. Environ. Microbiol. 62, 121-127. doi: 10.1128/aem.62.1.121-127.1996

Mawad, A. M. M., Abdel-Mageed, W. S., and Hesham, A. E. (2020). Quantification of naphthalene dioxygenase (NahAC) and catechol dioxygenase (C23O) catabolic genes produced by phenanthrenedegrading Pseudomonas fluorescens AH-40. Curr. Genomics 21, 111-118. doi: $10.2174 / 1389202921666200224101742$

Meng, Q.-W., Xu, Q.-Y., Zhu, T.-T., Jin, L., Fu, K.-Y., Guo, W.-C., et al. (2019). Hormonal signaling cascades required for phototaxis switch in wandering Leptinotarsa decemlineata larvae. PLoS Genet. 15:e1007423. doi: 10.1371/journal.pgen.1007423

Mushtaq, M. N., Sunohara, Y., and Matsumoto, H. (2013). Bioactive l-DOPA induced quinoprotein formation to inhibit root growth of cucumber seedlings. J. Pesticide Sci. 38, 68-73. doi: 10.1584/jpestics.D13-005

Nadkarni, M. A., Martin, F. E., Jacques, N. A., and Hunter, N. (2002). Determination of bacterial load by real-time PCR using a broadrange (universal) probe and primers set. Microbiology 148, 257-266. doi: 10.1099/00221287-148-1-257

O'Connor, K. E., Buckley, C. M., Hartmans, S., and Dobson, A. D. W. (1995). Possible regulatory role for non-aromatic carbon sources in styrene degradation by Pseudomonas putida CA-3. Appl. Environ. Microbiol. 61, 544-548. doi: 10.1128/aem.61.2.544-548.1995

O'Connor, K. E., and Dobson, A. D. W. (1996). Microbial degradation of alkenylbenzenes. World J. Microbiol. Biotechnol. 12, 207-212. doi: 10.1007/BF00360916

O'Leary, N. D., O'Connor, K. E., and Dobson, A. D. W. (2002). Biochemistry, genetics and physiology of microbial styrene degradation. FEMS Microbiol. Rev. 26, 403-417. doi: 10.1111/j.1574-6976.2002.tb00622.x

Panke, S., de Lorenzo, V., Kaiser, A., Witholt, B., and Wubbolts, M. G. (1999). Engineering of a stable whole-cell biocatalyst capable of (S)styrene oxide formation for continuous two-liquid-phase applications. Appl. Environ. Microbiol. 65, 5619-5623. doi: 10.1128/AEM.65.12.5619-5623. 1999 
Patel, K., and Patel, M. (2020). Improving bioremediation process of petroleum wastewater using biosurfactants producing Stenotrophomonas sp. S1VKR26 and assessment of phytotoxicity. Bioresour. Technol. 315:123861. doi: 10.1016/j.biortech.2020.123861

Podolak, I., Galanty, A., and Sobolewska, D. (2010). Saponins as cytotoxic agents: a review. Phytochem. Rev. 9, 425-474. doi: 10.1007/s11101-010-9183-Z

Pozdnyakova-Filatova, I., Petrikov, K., Vetrova, A., Frolova, A., Streletskii, R., and Zakharova, M. (2020). The naphthalene catabolic genes of Pseudomonas putida BS3701: additional regulatory control. Front. Microbiol. 11:1217. doi: $10.3389 /$ fmicb.2020.01217

Quesada, C. R., Scharf, M. E., and Sadof, C. S. (2020). Excretion of non-metabolized insecticides in honeydew of striped pine scale. Chemosphere 249:126167. doi: 10.1016/j.chemosphere.2020.126167

Rodgers, K. J., and Shiozawa, N. (2008). Misincorporation of amino acid analogues into proteins by biosynthesis. Int. J. Biochem. Cell Biol. 40, 1452-1466. doi: 10.1016/j.biocel.2008.01.009

Rozman, K. (1985). Intestinal excretion of toxic substances. Arch. Toxicol. Suppl. 8, 87-93. doi: 10.1007/978-3-642-69928-3 10

Shi, X.-Q., Guo, W.-C., Wan, P.-J., Zhou, L.-T., Ren, X.-L., Ahmat, T., et al. (2013). Validation of reference genes for expression analysis by quantitative real-time PCR in Leptinotarsa decemlineata (Say). BMC Res. Notes 6:93. doi: 10.1186/1756-0500-6-93

Silkie, S. S., and Nelson, K. L. (2009). Concentrations of host-specific and generic fecal markers measured by quantitative PCR in raw sewage and fresh animal feces. Water Res. 43, 4860-4871. doi: 10.1016/j.watres.2009.08.017

Steinmetz, Z., Kurtz, M. P., Zubrod, J. P., Meyer, A. H., Elsner, M., and Schaumann, G. E. (2019). Biodegradation and photooxidation of phenolic compounds in soil-A compound-specific stable isotope approach. Chemosphere 230, 210-218. doi: 10.1016/j.chemosphere.2019.05.030

Tachibana, S., Kuba, N., Kawai, F., Duine, J. A., and Yasuda, M. (2003). Involvement of a quinoprotein (PQQ-containing) alcohol dehydrogenase in the degradation of polypropylene glycols by the bacterium Stenotrophomonas maltophilia. FEMS Microbiol. Lett. 218, 345-349. doi: 10.1111/j.1574-6968.2003.tb11540.x

Utkin, I. B., Yakimov, M. M., Mateeva, L. N., Kozlyak, E. I., Rogozhin, I. S., Solomon, Z. G., et al. (1991). Degradation of styrene and ethylbenzene by Pseudomonas species Y2. FEMS Microbiol. Lett. 77, 237-242. doi: 10.1111/j.1574-6968.1991.tb04355.x

Van Dexter, S., and Boopathy, R. (2019). Biodegradation of phenol by Acinetobacter tandoii isolated from the gut of the termite. Environ. Sci. Pollut. Res. 26, 34067-33072. doi: 10.1007/s11356-018-3292-4

Velasco, A., Alonso, S., Garcia, J. L., Perera, J., and Diaz, E. (1998). Genetic and functional analysis of the styrene catabolic cluster of Pseudomonas sp. strain Y2. J. Bacteriol. 180, 1063-1071. doi: 10.1128/JB.180.5.1063-1071.1998
Vilanova, C., Baixeras, J., Latorre, A., and Porcar, M. (2016). The generalist inside the specialist: gut bacterial communities of two insect species feeding on toxic plants are dominated by Enterococcus sp. Front. Microbiol. 7:1005. doi: $10.3389 /$ fmicb. 2016.01005

Wu, Y., Zheng, Y., Chen, Y., Wang, S., Chen, Y., Hu, F., et al. (2020). Honey bee (Apis mellifera) gut microbiota promotes host endogenous detoxification capability via regulation of $\mathrm{P} 450$ gene expression in the digestive tract. Microb. Biotechnol. 13, 1201-1212. doi: 10.1111/1751-7915.13579

Xia, X., Lan, B., Tao, X., Lin, J., and You, M. (2020). Characterization of Spodoptera litura gut bacteria and their role in feeding and growth of the host. Front. Microbiol. 11:1492. doi: 10.3389/fmicb.2020.01492

Xiong, W., Yin, C., Peng, W., Deng, Z., Lin, S., and Liang, R. (2019). Characterization of an $17 \beta$-estradiol-degrading bacterium Stenotrophomonas maltophilia SJTL3 tolerant to adverse environmental factors. Appl. Microbiol. Biotechnol. 104, 1291-1305. doi: 10.1007/s00253-019-10281-8

Yen, K. M., and Gunsalus, I. C. (1982). Plasmid gene organization: naphthalene/salicylate oxidation. Proc. Natl. Acad. Sci. U.S.A. 79, 874-878. doi: 10.1073 /pnas.79.3.874

Zeng, J. Y., Wu, D. D., Shi, Z. B., Yang, J., Zhang, G. C., and Zhang, J. (2020). Influence of dietary aconitine and nicotine on the gut microbiota of two lepidopteran herbivores. Arch. Insect Biochem. Physiol. 23:e21676. doi: 10.1002/arch.21676

Zhang, S., Shu, J., Xue, H., Zhang, W., Zhang, Y., Liu, Y., et al. (2020). The gut microbiota in Camellia weevils are influenced by plant secondary metabolites and contribute to saponin degradation. mSystems 5, e00692e00619. doi: 10.1128/mSystems.00692-19

Conflict of Interest: The authors declare that the research was conducted in the absence of any commercial or financial relationships that could be construed as a potential conflict of interest.

Publisher's Note: All claims expressed in this article are solely those of the authors and do not necessarily represent those of their affiliated organizations, or those of the publisher, the editors and the reviewers. Any product that may be evaluated in this article, or claim that may be made by its manufacturer, is not guaranteed or endorsed by the publisher.

Copyright (C) 2021 Kang, Jin, Ma and Li. This is an open-access article distributed under the terms of the Creative Commons Attribution License (CC BY). The use, distribution or reproduction in other forums is permitted, provided the original author(s) and the copyright owner(s) are credited and that the original publication in this journal is cited, in accordance with accepted academic practice. No use, distribution or reproduction is permitted which does not comply with these terms. 